\title{
The ideal structure of semigroups of linear transformations with upper bounds on their nullity or defect
}

\author{
Suzana Mendes-Gonçalves ${ }^{\dagger}$ \\ Centro de Matemática, Universidade do Minho, 4710 Braga, Portugal \\ and \\ R P Sullivan \\ School of Mathematics \& Statistics \\ University of Western Australia, Nedlands 6009, Australia
}

\begin{abstract}
Suppose $V$ is a vector space with $\operatorname{dim} V=p \geq q \geq \aleph_{0}$, and let $T(V)$ denote the semigroup (under composition) of all linear transformations of $V$. For each $\alpha \in T(V)$, let ker $\alpha$ and $\operatorname{ran} \alpha$ denote the 'kernel' and the 'range' of $\alpha$, and write $n(\alpha)=\operatorname{dim} \operatorname{ker} \alpha$ and $d(\alpha)=\operatorname{codim} \operatorname{ran} \alpha$. In this paper, we study the semigroups $A M(p, q)=\{\alpha \in T(V): n(\alpha)<q\}$ and $A E(p, q)=\{\alpha \in$ $T(V): d(\alpha)<q\}$. First, we determine whether they belong to the class of all semigroups whose sets of bi-ideals and quasi-ideals coincide. Then, for each semigroup, we describe its maximal regular subsemigroup, and we characterise its Green's relations and (two-sided) ideals. As a precursor to further work in this area, we also determine all the maximal right simple subsemigroups of $A M(p, q)$.
\end{abstract}

AMS Primary Classification: 20M20; Secondary: 15A04.

Keywords: bi-ideal, quasi-ideal, linear transformation semigroup, maximal regular, maximal right simple

Proposed Running Head: Ideals in linear transformation semigroups

† Correspondence: Suzana Mendes-Gonçalves, Departamento de Matemática, Campus de Gualtar - Universidade do Minho, 4710-057 Braga, Portugal; E-mail: smendes@math.uminho.pt.

The authors acknowledge the support of the Portuguese Foundation for Science and Technology (FCT) through the research program POCTI. 


\section{Introduction}

By definition, every two-sided ideal of a semigroup is one-sided, and several authors have studied semigroups with the converse property: namely, every one-sided ideal is two-sided (that is, so-called duo semigroups: see [1] and the references therein). Likewise, it is worth studying semigroups with the BQ-property: namely, every biideal is a quasi-ideal. This idea first arose in [4] and it has been considered for various transformation semigroups (see [6] for a brief survey). Indeed, the notions of 'biideal' and 'quasi-ideal' date from over 30 years ago, and the significance of the latter was documented in [10]. In this paper, we consider the BQ-property and the ideal structure of certain linear transformation semigroups. However, to further explain the background to our work, we need some notation.

Let $X$ be an infinite set with cardinal $p$ and let $q$ be a cardinal such that $\aleph_{0} \leq q \leq p$. Let $T(X)$ denote the semigroup under composition of all (total) transformations from $X$ to $X$. If $\alpha \in T(X)$, we write ran $\alpha$ for the range of $\alpha$ and define the rank of $\alpha$ to be $r(\alpha)=|\operatorname{ran} \alpha|$. We also write

$$
\begin{array}{ll}
D(\alpha)=X \backslash \operatorname{ran} \alpha, & d(\alpha)=|D(\alpha)|, \\
C(\alpha)=\bigcup\left\{y \alpha^{-1}:\left|y \alpha^{-1}\right| \geq 2\right\}, & c(\alpha)=|C(\alpha)|,
\end{array}
$$

and refer to these cardinal numbers as the defect and the collapse of $\alpha$, respectively.

A transformation $\alpha \in T(X)$ is said to be almost one-to-one if $c(\alpha)$ is finite. By an almost onto transformation of $X$ we mean $\alpha \in T(X)$ such that $d(\alpha)$ is finite. In [5] Theorems 2.1 and 2.3, Kemprasit showed that $A M(X)$, the semigroup of all almost one-to-one transformations of $X$, and $A E(X)$, the semigroup of all almost onto transformations of $X$, do not belong to $\mathbf{B Q}$, the class of all semigroups whose sets of bi-ideals and quasi-ideals coincide (here, the notation ' $M$ ' signifies 'mono', and ' $E$ ' denotes 'epi').

Here, we examine related semigroups defined as follows. Let $V$ be a vector space over a field $F$ with dimension $p \geq \aleph_{0}$. Let $T(V)$ denote the semigroup (under composition) of all linear transformations from $V$ into itself. Also, let $M(V)$ denote the subsemigroup of $T(V)$ consisting of all one-to-one linear transformations, and let $E(V)$ denote the subsemigroup of $T(V)$ consisting of all onto linear transformations. If $\alpha \in T(V)$, we write ker $\alpha$ and $\operatorname{ran} \alpha$ for the kernel and the range of $\alpha$, and put

$$
n(\alpha)=\operatorname{dim} \operatorname{ker} \alpha, r(\alpha)=\operatorname{dim} \operatorname{ran} \alpha, d(\alpha)=\operatorname{codim} \operatorname{ran} \alpha .
$$

As usual, these are called the nullity, rank and defect of $\alpha$, respectively. For cardinals $q \leq p$, we write

$$
\begin{aligned}
& A M(p, q)=\{\alpha \in T(V): n(\alpha)<q\}, \quad \text { and } \\
& A E(p, q)=\{\alpha \in T(V): d(\alpha)<q\} .
\end{aligned}
$$

Clearly, $M(V) \subseteq A M(p, q)$ and $E(V) \subseteq A E(p, q)$. Because of Example 1 below, we will be interested only in the case that $q$ is infinite. Namnak and Kemprasit showed in [8] Theorems 2.2 and 2.3 that $A M\left(p, \aleph_{0}\right)$ and $A E\left(p, \aleph_{0}\right)$ do not belong to $\mathbf{B Q}$. In section 2, 
we generalise these results: we show that $A M(p, q)$ and $A E(p, q)$ are subsemigroups of $T(V)$; and we also show that they do not belong to BQ. For each of the two semigroups, we characterise its regular elements; and using this, we determine its unique maximal regular subsemigroup. In section 3, we characterise the Green's relations and ideals in $A M(p, q)$ and $A E(p, q)$ and in section 4 , we describe all the maximal right simple subsemigroups of $A M(p, q)$. In passing, we observe that Kemprasit and Namnak did not study Green's relations and ideals for any of the semigroups which they considered.

\section{Basic properties}

In what follows, $Y=A \cup \dot{\cup}$ means $Y$ is a disjoint union of $A$ and $B$, and we write $\operatorname{id}_{Y}$ for the identity transformation on $Y$.

As an abbreviation, we write $\left\{e_{i}\right\}$ to denote a subset $\left\{e_{i}: i \in I\right\}$ of $V$, taking as understood that the subscript $i$ belongs to some (unmentioned) index set $I$. The subspace $A$ of $V$ generated by a linearly independent subset $\left\{e_{i}\right\}$ of $V$ is denoted by $\left\langle e_{i}\right\rangle$, and then $\operatorname{dim} A=|I|$.

We adopt the convention introduced in [9]. That is, often it is necessary to define some $\alpha \in T(V)$ by first choosing a basis $\left\{e_{i}\right\}$ for $V$ and some $\left\{a_{i}\right\} \subseteq V$, and then letting $e_{i} \alpha=a_{i}$ for each $i$ and extending this action by linearity to the whole of $V$. To abbreviate matters, we simply say, given $\left\{e_{i}\right\}$ and $\left\{a_{i}\right\}$ within context, that $\alpha \in T(V)$ is defined by letting

$$
\alpha=\left(\begin{array}{c}
e_{i} \\
a_{i}
\end{array}\right)
$$

Often our argument starts by choosing a basis for ker $\alpha$ and expanding it to one for a subspace containing $\operatorname{ker} \alpha$ : provided no confusion will arise, we use this expression even if $\alpha$ is one-to-one (in which case, $\operatorname{ker} \alpha=\{0\}$ and so it has basis the empty set).

For every $\alpha, \beta \in T(V)$, we have $n(\alpha) \leq n(\alpha \beta)$ and $d(\beta) \leq d(\alpha \beta)$, since ker $\alpha \subseteq \operatorname{ker}(\alpha \beta)$ and $\operatorname{ran}(\alpha \beta) \subseteq \operatorname{ran} \beta$. The fact that the sets $A M(p, q)$ and $A E(p, q)$ are semigroups follows from parts (a) and (b), respectively, of the following result, and our assumption that $q$ is infinite. In effect, this result was proved by Namnak and Kemprasit in [8] pp. 217-218, but we include a brief proof for completeness.

Lemma 1. If $\alpha, \beta \in T(V)$ then

(a) $n(\alpha) \leq n(\alpha \beta) \leq n(\alpha)+n(\beta)$, and

(b) $d(\beta) \leq d(\alpha \beta) \leq d(\alpha)+d(\beta)$.

Proof. Let $\alpha, \beta \in T(V)$ and recall that $(\operatorname{ker}(\alpha \beta)) \alpha=\operatorname{ker} \beta \cap \operatorname{ran} \alpha$. If $\operatorname{ker}(\alpha \beta)=\operatorname{ker} \alpha \oplus$ $\left\langle e_{j}\right\rangle$ then $(\operatorname{ker}(\alpha \beta)) \alpha=\left\langle e_{j} \alpha\right\rangle \subseteq \operatorname{ker} \beta$, so $|J| \leq n(\beta)$ and hence $n(\alpha \beta)=n(\alpha)+|J| \leq$ $n(\alpha)+n(\beta)$. Now suppose $\operatorname{ran} \beta=\operatorname{ran}(\alpha \beta) \oplus\left\langle e_{i}\right\rangle$. Then $d(\alpha \beta)=d(\beta)+|I|$, where $|I|=\operatorname{dim}(\operatorname{ran} \beta / \operatorname{ran}(\alpha \beta))$. Clearly if $V=(\operatorname{ran} \alpha+\operatorname{ker} \beta) \oplus U$, then $d(\alpha) \geq \operatorname{dim} U$ and $\operatorname{ran} \beta=\operatorname{ran}(\alpha \beta) \oplus U \beta$ (for, if $w=v \alpha \beta=u \beta$ then $v \alpha-u \in \operatorname{ker} \beta$, so $u \in \operatorname{ran} \alpha+\operatorname{ker} \beta$ and this implies $u=0$, so $w=0)$. Hence $\operatorname{dim}(\operatorname{ran} \beta / \operatorname{ran}(\alpha \beta))=\operatorname{dim}(U \beta) \leq \operatorname{dim} U \leq$ $\operatorname{dim}(V / \operatorname{ran} \alpha)=d(\alpha)$, and the result follows.

Example 1. We note that $A M(p, q)$ and $A E(p, q)$ are semigroups only when $q$ is infinite (or 1). For, suppose $q$ is finite, $q \neq 1$, and let $\left\{e_{i}\right\} \dot{\cup}\left\{u_{1}, u_{2}, \ldots, u_{q}\right\}$ be a basis 
for $V$, with $|I|=p$. Now define $\alpha, \beta \in T(V)$ by

$$
\alpha=\left(\begin{array}{ccccc}
u_{1} & u_{2} & \ldots & u_{q} & e_{i} \\
0 & u_{2} & \ldots & u_{q} & e_{i}
\end{array}\right), \quad \beta=\left(\begin{array}{ccccc}
u_{1} & u_{2} & \ldots & u_{q} & e_{i} \\
u_{1} & 0 & \ldots & 0 & e_{i}
\end{array}\right) .
$$

Clearly, $n(\alpha)=d(\alpha)=1$ and $n(\beta)=d(\beta)=q-1$, and so $\alpha, \beta \in A M(p, q) \cap A E(p, q)$. It is easy to see that $\operatorname{ker}(\alpha \beta)=\left\langle u_{1}, u_{2}, \ldots, u_{q}\right\rangle$ and $V=\operatorname{ran}(\alpha \beta) \oplus\left\langle u_{1}, u_{2}, \ldots, u_{q}\right\rangle$. Therefore, $n(\alpha \beta)=d(\alpha \beta)=q$ and hence $\alpha \beta \notin A M(p, q) \cup A E(p, q)$.

A subsemigroup $Q$ of a semigroup $S$ is called a quasi-ideal of $S$ if $S Q \cap Q S \subseteq Q$. A subsemigroup $B$ of $S$ is a bi-ideal of $S$ if $B S B \subseteq B$. Note that every right and every left ideal of $S$ is a quasi-ideal, and every quasi-ideal $Q$ of a semigroup $S$ is a bi-ideal of $S$ since $Q S Q \subseteq S Q \cap Q S$. Given a non-empty subset $X$ of $S$, the quasiideal and the bi-ideal generated by $X$ will be denoted respectively by $(X)_{Q}$ and $(X)_{B}$. If $X=\left\{x_{1}, x_{2}, \ldots, x_{n}\right\}$ then we write $\left(x_{1}, x_{2}, \ldots, x_{n}\right)_{Q}$ and $\left(x_{1}, x_{2}, \ldots, x_{n}\right)_{B}$ instead of $\left(\left\{x_{1}, x_{2}, \ldots, x_{n}\right\}\right)_{Q}$ and $\left(\left\{x_{1}, x_{2}, \ldots, x_{n}\right\}\right)_{B}$, respectively. By [2] Vol. 1, pp. 84-85, Exercises 15 and 17, if $X$ is a non-empty subset of a semigroup $S$, then

$$
\begin{aligned}
& (X)_{Q}=S^{1} X \cap X S^{1}=(S X \cap X S) \cup X, \text { and } \\
& (X)_{B}=\left(X S^{1} X\right) \cup X=X S X \cup X \cup X^{2} .
\end{aligned}
$$

It is known that regular semigroups, right [left] simple semigroups and right [left] 0simple semigroups are in the class BQ of all semigroups whose sets of bi-ideals and quasi-ideals coincide (see [8] Propositions 1.2 and 1.3 for references to these results). On the other hand, by [8] Corollary 1.5, if $(x)_{B} \neq(x)_{Q}$ for some element $x$ of a semigroup $S$, then $S \notin \mathbf{B Q}$.

We now decide whether $A M(p, q)$ belongs to BQ. For this, we follow the argument for [8] Theorem 2.2, although the latter concerned only the case $q=\aleph_{0}$.

Theorem 1. For any infinite cardinals $p \geq q$, the semigroup $A M(p, q)$ does not belong to $\mathbf{B Q}$.

Proof. Suppose $\left\{e_{i}\right\}$ is a basis for $V$ and write $\left\{e_{i}\right\}=\left\{f_{i}\right\} \dot{\cup}\left\{f_{j}\right\}$ with $|J|=q$. Now write $\left\{f_{j}\right\}=\left\{a_{j}\right\} \dot{\cup}\left\{b_{k}\right\}$ with $|K|<q$ and $\left\{a_{j}\right\}=\left\{g_{j}\right\} \dot{\cup}\left\{h_{j}\right\}$. Put $\left\{h_{j}\right\} \dot{\cup}\left\{b_{k}\right\}=\left\{c_{j}\right\}$ and define $\alpha, \beta \in T(V)$ by

$$
\alpha=\left(\begin{array}{cc}
f_{i} & f_{j} \\
f_{i} & g_{j}
\end{array}\right), \quad \beta=\left(\begin{array}{cc}
f_{i} & f_{j} \\
f_{i} & a_{j}
\end{array}\right) .
$$

Since $n(\alpha)=0=n(\beta)$, we have $\alpha, \beta \in A M(p, q)$. Now define $\gamma \in T(V)$ by

$$
\gamma=\left(\begin{array}{cccc}
f_{i} & g_{j} & h_{j} & b_{k} \\
f_{i} & a_{j} \alpha & h_{j} & b_{k}
\end{array}\right)
$$

Since $\left\{a_{j} \alpha\right\} \subseteq\left\{f_{j} \alpha\right\}=\left\{g_{j}\right\}$, it follows that $\gamma$ is one-to-one and so $\gamma \in A M(p, q)$. Clearly, $\beta \alpha=\alpha \gamma$ and hence $\beta \alpha \in A M(p, q) \alpha \cap \alpha A M(p, q)=(\alpha)_{Q}$ (the intersection contains $\alpha$ since $A M(p, q)$ contains $\left.\operatorname{id}_{V}\right)$.

Suppose $\beta \alpha \in(\alpha)_{B}$. Then, $\beta \alpha \in \alpha A M(p, q) \alpha \cup\{\alpha\}$ (again, note that $A M(p, q)$ contains $\mathrm{id}_{V}$, so the first set in this union contains $\alpha^{2}$ ). If $\beta \alpha=\alpha$ then, since $\alpha$ 
is one-to-one, $\beta=\mathrm{id}_{V}$, a contradiction. Thus, there exists $\lambda \in A M(p, q)$ such that $\beta \alpha=\alpha \lambda \alpha$. Since $\alpha$ is one-to-one, it follows that $\beta=\alpha \lambda$. Hence, $\left\langle f_{i}, a_{j}\right\rangle=\operatorname{ran} \beta=$ $\operatorname{ran}(\alpha \lambda)=(\operatorname{ran} \alpha) \lambda=\left\langle f_{i}, g_{j}\right\rangle \lambda$ and so $V=\left\langle f_{i}, a_{j}, b_{k}\right\rangle=\left\langle f_{i}, g_{j}\right\rangle \lambda+\left\langle b_{k}\right\rangle$. For each $j, c_{j} \lambda \in V$, and so there exist $u_{j} \in\left\langle f_{i}, g_{j}\right\rangle$ and $v_{j} \in\left\langle b_{k}\right\rangle$ such that $c_{j} \lambda=u_{j} \lambda+v_{j}$. Then, $\left(c_{j}-u_{j}\right) \lambda=v_{j} \in\left\langle b_{k}\right\rangle$. Since $\left\{c_{j}\right\} \dot{\cup}\left\{f_{i}\right\} \dot{\cup}\left\{g_{j}\right\}$ is linearly independent, it follows that $\left\{c_{j}-u_{j}\right\}$ is also linearly independent and $c_{r}-u_{r} \neq c_{s}-u_{s}$ if $r \neq s$. Let $C=\left\langle c_{j}-u_{j}\right\rangle$. Then, $\operatorname{dim} C=q$ and $\operatorname{ran}(\lambda \mid C) \subseteq\left\langle b_{k}\right\rangle$. Hence, $\operatorname{dim}(\operatorname{ran}(\lambda \mid C))<q$. Since $q=\operatorname{dim} C=\operatorname{dim}(\operatorname{ker}(\lambda \mid C))+\operatorname{dim}(\operatorname{ran}(\lambda \mid C))$ by the Rank-Nullity Theorem, it follows that $\operatorname{dim}(\operatorname{ker}(\lambda \mid C))=q$. But $\operatorname{ker}(\lambda \mid C) \subseteq \operatorname{ker} \lambda$ and so $n(\lambda) \geq n(\lambda \mid C)=q$, which contradicts the fact that $\lambda \in A M(p, q)$. Therefore, $\beta \alpha \notin(\alpha)_{B}$ and so $(\alpha)_{Q} \neq(\alpha)_{B}$. By [8] Corollary 1.5, $A M(p, q) \notin \mathbf{B Q}$.

From a remark before Theorem 1, it follows that the semigroup $A M(p, q)$ is neither regular nor right simple nor left simple, for any infinite cardinals $p, q$ such that $p \geq q$. Hence, it is worth determining all regular elements in $A M(p, q)$.

Theorem 2. Let $\alpha \in A M(p, q)$. Then, $\alpha$ is regular if and only if $\alpha \in A E(p, q)$. Consequently, $A M(p, q) \cap A E(p, q)$ is the largest regular subsemigroup of $A M(p, q)$.

Proof. Suppose $\alpha \in A E(p, q)$. Let $\left\{e_{j}\right\}$ be a basis for ker $\alpha$ and expand it to a basis $\left\{e_{j}\right\} \cup \dot{\cup}\left\{e_{i}\right\}$ for $V$. Now write $e_{i} \alpha=a_{i}$ for each $i$. Since $\left\{a_{i}\right\}$ is a basis for ran $\alpha$, it can be expanded to a basis for $V$, say $\left\{a_{i}\right\} \cup \dot{\cup}\left\{a_{k}\right\}$. Define $\beta \in T(V)$ by

$$
\beta=\left(\begin{array}{cc}
a_{i} & a_{k} \\
e_{i} & 0
\end{array}\right) \text {. }
$$

Clearly, $n(\beta)=d(\alpha)<q$ and $d(\beta)=n(\alpha)<q$, and hence $\beta \in A M(p, q) \cap A E(p, q)$. Also, $\alpha=\alpha \beta \alpha$ and so $\alpha$ is regular in $A M(p, q)$. Conversely, suppose $\alpha=\alpha \beta \alpha$ for some $\beta \in A M(p, q)$. Then $\beta \alpha$ is an idempotent in $T(V)$, so $V=\operatorname{ker}(\beta \alpha) \oplus \operatorname{ran}(\beta \alpha)$ and, since $A M(p, q)$ is closed, it follows that $q>n(\beta \alpha)=d(\beta \alpha) \geq d(\alpha)$. Therefore, $\alpha \in A E(p, q)$ as required.

Finally, given a regular subsemigroup $S$ of $A M(p, q)$, we know it is contained in $A E(p, q)$, and so $S \subseteq A M(p, q) \cap A E(p, q)$. Thus, the latter is the largest regular subsemigroup of $A M(p, q)$.

Similar results hold for the semigroup $A E(p, q)$, as we now proceed to show. In the proof of our next theorem, we use an argument similar to the one used in [8] Theorem 2.3 , but ours is complicated by the possibility that $q>\aleph_{0}$.

Theorem 3. For any infinite cardinals $p \geq q$, the semigroup $A E(p, q)$ does not belong to $\mathbf{B Q}$.

Proof. Suppose $\left\{e_{i}\right\}$ is a basis for $V$ and write $\left\{e_{i}\right\}=\left\{f_{i}\right\} \dot{\cup}\{h\}$. Now write $\left\{f_{i}\right\}=$ $\left\{a_{i}\right\} \dot{\cup}\left\{b_{i}\right\}$ and define $\alpha, \beta \in T(V)$ by

$$
\alpha=\left(\begin{array}{ccc}
a_{i} & b_{i} & h \\
f_{i} & 0 & h
\end{array}\right), \quad \beta=\left(\begin{array}{ccc}
a_{i} & b_{i} & h \\
a_{i} & b_{i} & 0
\end{array}\right) .
$$

Since $d(\alpha)=0$ and $d(\beta)=\operatorname{dim}\langle h\rangle=1<q$, we have $\alpha, \beta \in A E(p, q)$. Also, $\alpha \neq \beta \alpha=$ $\alpha \beta$ and so $\alpha \beta \in A E(p, q) \alpha \cap \alpha A E(p, q)=(\alpha)_{Q}$ (note that the intersection contains $\alpha$, since $A E(p, q)$ contains $\left.\mathrm{id}_{V}\right)$. 
Now suppose $\alpha \beta \in(\alpha)_{B}=\alpha A E(p, q) \alpha \cup\{\alpha\}$ (again, note that $A E(p, q)$ contains $\operatorname{id}_{V}$, and so the first set in this union contains $\left.\alpha^{2}\right)$. Then, since $\alpha \beta \neq \alpha$, we know $\alpha \beta=\alpha \lambda \alpha$ for some $\lambda \in A E(p, q)$ and the surjectivity of $\alpha$ implies $\beta=\lambda \alpha$. Thus, $(h \lambda) \alpha=h(\lambda \alpha)=h \beta=0$ and so $h \lambda \in \operatorname{ker} \alpha$. Hence, there exist a natural number $n$ and scalars $x_{1}, \ldots, x_{n}$ such that

$$
h \lambda=\sum_{r=1}^{n} x_{r} b_{i_{r}}
$$

Put $\left\{b_{i}\right\} \backslash\left\{b_{i_{1}}, \ldots, b_{i_{n}}\right\}=\left\{c_{i}\right\}$. We assert that $\left\{c_{i}+\operatorname{ran} \lambda\right\}$ is a linearly independent subset of $V / \operatorname{ran} \lambda$. Suppose $\sum y_{i}\left(c_{i}+\operatorname{ran} \lambda\right)=\operatorname{ran} \lambda$ for some scalars $y_{i}$. Then, $\sum y_{i} c_{i} \in$ $\operatorname{ran} \lambda$ and so there exists some $u \in V$ such that $\sum y_{i} c_{i}=u \lambda$. Since $V=\left\langle a_{i}\right\rangle \oplus\left\langle b_{i}\right\rangle \oplus\langle h\rangle$, there exist scalars $r_{i}$ and $s$, and a vector $v \in\left\langle a_{i}\right\rangle$, such that $u=v+\sum r_{i} b_{i}+s h$. Hence,

$$
\sum y_{i} c_{i}=v \lambda+\sum r_{i}\left(b_{i} \lambda\right)+s(h \lambda) .
$$

Thus,

$$
\sum y_{i}\left(c_{i} \alpha\right)=v(\lambda \alpha)+\sum r_{i}\left(b_{i} \lambda \alpha\right)+s(h \lambda \alpha) .
$$

Since $\operatorname{ker} \alpha=\left\langle b_{i}\right\rangle, \lambda \alpha=\beta$ and $\operatorname{ker} \beta=\langle h\rangle$, it follows that $0=v \beta+\sum r_{i}\left(b_{i} \beta\right)$. That is, $v+\sum r_{i} b_{i} \in \operatorname{ker} \beta$ and, by our choice of bases, this implies $v=0$ and $r_{i}=0$ for each $i$. Thus, we can rewrite (2):

$$
\sum y_{i} c_{i}=s(h \lambda)
$$

From (1),

$$
\sum y_{i} c_{i}=\sum_{r=1}^{n}\left(s x_{r}\right) b_{i_{r}}
$$

Since $\left\{c_{i}\right\} \dot{\cup}\left\{b_{i_{1}}, \ldots, b_{i_{n}}\right\}$ is linearly independent, it follows that $y_{i}=0$ for each $i$. Hence, $\left\{c_{i}+\operatorname{ran} \lambda\right\}$ is linearly independent, and so $q>d(\lambda)=\operatorname{dim}(V / \operatorname{ran} \lambda)=p$, a contradiction. Therefore, $\alpha \beta \notin(\alpha)_{B}$ and so $(\alpha)_{B} \neq(\alpha)_{Q}$. Hence, by [8] Corollary 1.5, $A E(p, q) \notin \mathbf{B Q}$.

From the previous Theorem, it follows that $A E(p, q)$ is neither regular nor right simple or left simple, for any infinite cardinals $p$ and $q$ such that $p \geq q$. In the next result, we determine all regular elements in $A E(p, q)$.

Theorem 4. Let $\alpha \in A E(p, q)$. Then, $\alpha$ is regular if and only if $\alpha \in A M(p, q)$. Consequently, $A M(p, q) \cap A E(p, q)$ is the largest regular subsemigroup of $A E(p, q)$.

Proof. By Theorem 2, if $\alpha \in A M(p, q)$ then $\alpha=\alpha \beta \alpha$ and $\beta=\beta \alpha \beta$ for some $\beta \in$ $A M(p, q)$, and hence $\beta \in A E(p, q)$ (by Theorem 2 again). That is, every $\alpha \in A M(p, q) \cap$ $A E(p, q)$ is a regular element of $A E(p, q)$. Conversely, suppose $\alpha \in A E(p, q)$ and $\alpha=\alpha \beta \alpha$ for some $\beta \in A E(p, q)$. Then $\alpha \beta$ is an idempotent in $T(V)$, and hence $V=\operatorname{ker}(\alpha \beta) \oplus \operatorname{ran}(\alpha \beta)$ and, since $A E(p, q)$ is closed, it follows that $q>d(\alpha \beta)=$ $n(\alpha \beta) \geq n(\alpha)$. Therefore, $\alpha \in A M(p, q)$ as required. Finally, as in the last paragraph of the proof of Theorem 2, $A M(p, q) \cap A E(p, q)$ is the largest regular subsemigroup of $A E(p, q)$. 


\section{Green's relations and ideals}

Green's relations on $T(V)$ are well-known: if $\alpha, \beta \in T(V)$, then $\alpha \mathcal{L} \beta$ if and only if $\operatorname{ran} \alpha=\operatorname{ran} \beta ; \alpha \mathcal{R} \beta$ if and only if $\operatorname{ker} \alpha=\operatorname{ker} \beta$; and $\mathcal{D}=\mathcal{J}$ [2] Vol. 1, Exercise 2.2.6. Moreover, by Hall's Theorem ([3], Proposition II.4.5), any regular subsemigroup of $T(V)$ inherits characterisations of its Green's relations from those on $T(V)$. From section 2, we know $A M(p, q)$ and $A E(p, q)$ are not regular, so it is surprising that, nonetheless, the $\mathcal{L}$-relation on $A M(p, q)$ and the $\mathcal{R}$-relation on $A E(p, q)$ can be described just like the corresponding ones on $T(V)$, and moreover $\mathcal{D}=\mathcal{J}$ for both semigroups. On the other hand, their ideal structure differs markedly from that of $T(V)$, as we eventually show in this section.

First, we characterise the $\mathcal{L}$ relation on $A M(p, q)$ and the $\mathcal{R}$ relation on $A E(p, q)$.

Lemma 2. Let $\alpha, \beta \in A M(p, q)$. Then $\alpha \mathcal{L} \beta$ if and only if $\operatorname{ran} \alpha=\operatorname{ran} \beta$.

Proof. Suppose $\operatorname{ran} \alpha=\operatorname{ran} \beta$ and let $\left\{e_{j}\right\}$ be a basis for $\operatorname{ker} \beta$. Expand $\left\{e_{j}\right\}$ to a basis $\left\{e_{j}\right\} \cup\left\{e_{i}\right\}$ for $V$ and write $e_{i} \beta=b_{i}$ for each $i$. Then, $\left\{b_{i}\right\}$ is a basis for $\operatorname{ran} \beta=\operatorname{ran} \alpha$. For every $i$, choose $f_{i} \in b_{i} \alpha^{-1}$. Clearly, $\left\{f_{i}\right\}$ is linearly independent. Now define $\lambda \in T(V)$ by

$$
\lambda=\left(\begin{array}{cc}
e_{j} & e_{i} \\
0 & f_{i}
\end{array}\right) .
$$

Since $\operatorname{ker} \lambda=\operatorname{ker} \beta$, it follows that $\lambda \in A M(p, q)$. Also, $\beta=\lambda \alpha$. Similarly, we conclude that there exists $\mu \in A M(p, q)$ such that $\alpha=\mu \beta$, and so $\alpha \mathcal{L} \beta$. The converse involves a standard argument, so we omit the details.

Lemma 3. Let $\alpha, \beta \in A E(p, q)$. Then $\alpha \mathcal{R} \beta$ if and only if $\operatorname{ker} \alpha=\operatorname{ker} \beta$.

Proof. Suppose ker $\alpha=\operatorname{ker} \beta$ and let $\left\{e_{j}\right\}$ be a basis for this subspace. Expand $\left\{e_{j}\right\}$ to a basis $\left\{e_{j}\right\} \cup\left\{e_{i}\right\}$ for $V$ and, for each $i$, write $e_{i} \alpha=a_{i}$ and $e_{i} \beta=b_{i}$. Clearly, $\left\{a_{i}\right\}$ and $\left\{b_{i}\right\}$ are bases for $\operatorname{ran} \alpha$ and $\operatorname{ran} \beta$, respectively. Now expand $\left\{b_{i}\right\}$ to a basis for $V$, say $\left\{b_{i}\right\} \dot{\cup}\left\{b_{\ell}\right\}$, and define $\lambda \in T(V)$ by

$$
\lambda=\left(\begin{array}{cc}
b_{\ell} & b_{i} \\
0 & a_{i}
\end{array}\right) .
$$

Since $d(\lambda)=d(\alpha)$, it follows that $\lambda \in A E(p, q)$. Also, $\alpha=\beta \lambda$. Similarly we conclude that there exists $\mu \in A E(p, q)$ such that $\beta=\alpha \mu$. Hence $\alpha \mathcal{R} \beta$. The converse involves a standard argument, so we omit the details.

We proceed to characterise the $\mathcal{R}$ relation on $A M(p, q)$. For this, we need two preliminary Lemmas.

Lemma 4. If $\alpha, \beta, \lambda \in T(V)$ satisfy $\alpha=\beta \lambda$ then

$$
d(\beta) \leq n(\lambda)+\operatorname{dim}(\operatorname{ran} \lambda / \operatorname{ran} \alpha) .
$$

In fact, if we also have $\operatorname{ker} \alpha=\operatorname{ker} \beta$, then $d(\beta)=n(\lambda)+\operatorname{dim}(\operatorname{ran} \lambda / \operatorname{ran} \alpha)$.

Proof. Since $\alpha=\beta \lambda$ implies $\operatorname{ker} \beta \subseteq \operatorname{ker} \alpha$, we can write $\operatorname{ker} \beta=\left\langle e_{r}\right\rangle$, $\operatorname{ker} \alpha=\left\langle e_{r}, e_{s}\right\rangle$ and $V=\left\langle e_{r}\right\rangle \oplus\left\langle e_{s}\right\rangle \oplus\left\langle e_{j}\right\rangle$. Write $e_{j} \alpha=a_{j}, e_{s} \beta=b_{s}$ and $e_{j} \beta=b_{j}$, and note that $a_{j}=e_{j} \alpha=\left(e_{j} \beta\right) \lambda=b_{j} \lambda$ for each $j$. In addition, $\left\{a_{j}\right\}$ and $\left\{b_{s}, b_{j}\right\}$ are bases for $\operatorname{ran} \alpha$ 
and $\operatorname{ran} \beta$, respectively. Now, if $\sum x_{j} b_{j} \in \operatorname{ker} \lambda$ for some scalars $x_{j}$, then $\sum x_{j} a_{j}=0$ and so $x_{j}=0$ for each $j$ : that is, $\left\langle b_{j}\right\rangle \cap \operatorname{ker} \lambda=\{0\}$. Hence we can write $V=\left\langle b_{j}\right\rangle \oplus \operatorname{ker} \lambda \oplus\left\langle e_{k}\right\rangle$ and we assert that $\operatorname{ran} \lambda=\operatorname{ran} \alpha \oplus\left\langle e_{k} \lambda\right\rangle$. For, if $\sum x_{j} a_{j}=\sum y_{k}\left(e_{k} \lambda\right)$ for some scalars $x_{j}$ and $y_{k}$ then $\sum x_{j} b_{j}-\sum y_{k} e_{k} \in \operatorname{ker} \lambda$ and, by our choice of bases, this implies $x_{j}=0=y_{k}$ for all $j$ and $k$. Clearly, $\left\{e_{k} \lambda\right\}$ is linearly independent. Since $\left\langle b_{j}\right\rangle \subseteq \operatorname{ran} \beta$, we have

$$
d(\beta) \leq \operatorname{codim}\left\langle b_{j}\right\rangle=n(\lambda)+|K|=n(\lambda)+\operatorname{dim}(\operatorname{ran} \lambda / \operatorname{ran} \alpha) .
$$

Finally, if we also have $\operatorname{ker} \alpha=\operatorname{ker} \beta$ then, with the previous notation, $\operatorname{ran} \beta=\left\langle b_{j}\right\rangle$ and $V=\left\langle b_{j}\right\rangle \oplus \operatorname{ker} \lambda \oplus\left\langle e_{k}\right\rangle$ and so $d(\beta)=n(\lambda)+|K|$.

Lemma 5. If $\alpha, \beta \in A M(p, q)$ and $\alpha \mathcal{R} \beta$, then $\alpha \in A E(p, q)$ if and only if $\beta \in$ $A E(p, q)$.

Proof. Suppose the conditions hold and $\alpha \in A E(p, q)$. By Theorem 2, $\alpha$ is a regular element of $A M(p, q)$, and so $D_{\alpha}$, the $\mathcal{D}$-class of $\alpha$ in $A M(p, q)$, is regular (by [2] Vol. 1, Theorem 2.11). Now let $R_{\alpha}$ denote the $\mathcal{R}$-class of $\alpha$ in $A M(p, q)$. Since $\beta \in R_{\alpha} \subseteq D_{\alpha}$, this implies $\beta$ is a regular element of $A M(p, q)$ and so $\beta \in A E(p, q)$ by Theorem 2 . Similarly, if $\beta \in A E(p, q)$ then $\alpha \in A E(p, q)$.

Lemma 6. Let $\alpha \in A M(p, q)$ and denote the $\mathcal{R}$-class of $A M(p, q)$ containing $\alpha$ by $R_{\alpha}$. Then,

(a) $\alpha \in A E(p, q)$ implies $R_{\alpha}=\{\beta \in A M(p, q): \beta \in A E(p, q)$ and $\operatorname{ker} \beta=\operatorname{ker} \alpha\}$;

(b) $\alpha \notin A E(p, q)$ implies $R_{\alpha}=\{\beta \in A M(p, q): \operatorname{ker} \beta=\operatorname{ker} \alpha$ and $d(\beta)=d(\alpha)\}$.

Proof. First suppose $\alpha \in A E(p, q)$. If $\beta \in A M(p, q)$ is such that $\alpha \mathcal{R} \beta$, then, since $\operatorname{id}_{V} \in A M(p, q)$, there exist $\lambda, \mu \in A M(p, q)$ such that $\alpha=\beta \lambda$ and $\beta=\alpha \mu$. Therefore ker $\alpha=\operatorname{ker} \beta$. Also, we know $\beta \in A E(p, q)$, from Lemma 5 .

Conversely, suppose $\beta \in A M(p, q) \cap A E(p, q)$ and $\operatorname{ker} \beta=\operatorname{ker} \alpha$. Since $A M(p, q) \cap$ $A E(p, q)$ is a regular subsemigroup of $A E(p, q)$, Hall's Theorem ([3], Proposition II.4.5) implies that the $\mathcal{R}$ relation on $A M(p, q) \cap A E(p, q)$ is the restriction of the $\mathcal{R}$ relation on $A E(p, q)$ to $A M(p, q) \cap A E(p, q)$. In other words, since $\alpha, \beta \in A M(p, q) \cap A E(p, q)$ and $\operatorname{ker} \alpha=\operatorname{ker} \beta$, we deduce from Lemma 3 that $\alpha \mathcal{R} \beta$ in $A M(p, q) \cap A E(p, q)$ and hence $\alpha \mathcal{R} \beta$ in $A M(p, q)$. That is, $\beta \in R_{\alpha}$ as required, and (a) holds.

Now, suppose $\alpha \notin A E(p, q)$ and $\alpha \mathcal{R} \beta$ in $A M(p, q)$. Then $\beta \notin A E(p, q)$ (by Lemma 5) and $\alpha=\beta \lambda, \beta=\alpha \mu$ for some $\lambda, \mu \in A M(p, q)$. As we already know, the latter implies $\operatorname{ker} \alpha=\operatorname{ker} \beta$. Moreover, since $\alpha=\beta \lambda, n(\lambda)<q$ and $d(\beta) \geq q$, by Lemma 4 we have $d(\beta) \leq \operatorname{dim}(\operatorname{ran} \lambda / \operatorname{ran} \alpha) \leq \operatorname{dim}(V / \operatorname{ran} \alpha)=d(\alpha)$. Similarly, since $\beta=\alpha \mu, n(\mu)<q$ and $d(\alpha) \geq q$, we deduce that $d(\alpha) \leq d(\beta)$ and equality follows.

Conversely, suppose $\beta \in A M(p, q)$ is such that $\operatorname{ker} \beta=\operatorname{ker} \alpha$ and $d(\beta)=d(\alpha)$. Let $\left\{e_{j}\right\}$ be a basis for $\operatorname{ker} \alpha=\operatorname{ker} \beta$, with $|J|=n(\alpha)=n(\beta)$, and expand it to a basis $\left\{e_{j}\right\} \cup \dot{\cup}\left\{e_{i}\right\}$ for $V$. Now write $e_{i} \alpha=a_{i}$ and $e_{i} \beta=b_{i}$ for each $i$. Then, $\left\{a_{i}\right\}$ is a basis for ran $\alpha$ and it can be expanded to a basis for $V$, say $\left\{a_{i}\right\} \cup \dot{\cup}\left\{a_{k}\right\}$, where $|K|=d(\alpha) \geq q$. Similarly, $\left\{b_{i}\right\}$ is a basis for $\operatorname{ran} \beta$ and we can expand it to a basis $\left\{b_{i}\right\} \dot{\cup}\left\{b_{k}\right\}$ for $V$ (note that $d(\beta)=d(\alpha)=|K|$ ). Since $|K| \geq q$, we can write $\left\{a_{k}\right\}$ as $\left\{u_{k}\right\} \dot{\cup}\left\{u_{r}\right\}$ and $\left\{b_{k}\right\}$ as $\left\{v_{k}\right\} \dot{\cup}\left\{v_{r}\right\}$, where $|R|<q$. Now define $\lambda, \mu \in T(V)$ by

$$
\lambda=\left(\begin{array}{ccc}
b_{i} & v_{k} & v_{r} \\
a_{i} & u_{k} & 0
\end{array}\right), \quad \mu=\left(\begin{array}{ccc}
a_{i} & u_{k} & u_{r} \\
b_{i} & v_{k} & 0
\end{array}\right) .
$$


Since $n(\lambda)=\operatorname{dim}\left\langle v_{r}\right\rangle<q$ and $n(\mu)=\operatorname{dim}\left\langle u_{r}\right\rangle<q$, we have $\lambda, \mu \in A M(p, q)$. Also, $\alpha=\beta \lambda$ and $\beta=\alpha \mu$. Hence, $\alpha \mathcal{R} \beta$ and (b) holds.

The next two results are crucial for the characterisation of the $\mathcal{L}$ relation on $A E(p, q)$.

Lemma 7. If $\alpha, \beta, \lambda \in T(V)$ satisfy $\alpha=\lambda \beta$, then

$$
n(\beta) \leq d(\lambda)+\operatorname{dim}(\operatorname{ker} \alpha / \operatorname{ker} \lambda) .
$$

In fact, if $\operatorname{ran} \alpha=\operatorname{ran} \beta$ then $n(\beta)=d(\lambda)+\operatorname{dim}(\operatorname{ker} \alpha / \operatorname{ker} \lambda)$.

Proof. Since $\alpha=\lambda \beta$, we can write $\operatorname{ker} \lambda=\left\langle e_{j}\right\rangle$, $\operatorname{ker} \alpha=\left\langle e_{j}\right\rangle \oplus\left\langle e_{i}\right\rangle$ and $V=\left\langle e_{j}\right\rangle \oplus$ $\left\langle e_{i}\right\rangle \oplus\left\langle f_{k}\right\rangle$. Write $f_{k} \alpha=a_{k}$ and $f_{k} \lambda=u_{k}$ for each $k$, and note that $\left\{a_{k}\right\}$ is a basis for $\operatorname{ran} \alpha$. In addition, $a_{k}=f_{k} \alpha=u_{k} \beta$. Clearly, the set $\left\{e_{i} \lambda\right\} \cup \dot{\cup}\left\{u_{k}\right\}$ is linearly independent, and hence $\operatorname{ran} \lambda=\left\langle e_{i} \lambda\right\rangle \oplus\left\langle u_{k}\right\rangle$. Moreover, if $\left(\sum x_{k} u_{k}\right) \beta=0$ for some scalars $x_{k}$, then $\sum x_{k}\left(u_{k} \beta\right)=0$, and hence $\sum x_{k} a_{k}=0$ and so $x_{k}=0$ for each $k$. Thus $\operatorname{ker} \beta \cap\left\langle u_{k}\right\rangle=\{0\}$. Therefore,

$$
n(\beta) \leq \operatorname{codim}\left\langle u_{k}\right\rangle=d(\lambda)+|I|=d(\lambda)+\operatorname{dim}(\operatorname{ker} \alpha / \operatorname{ker} \lambda) .
$$

Now suppose $\operatorname{ran} \beta=\operatorname{ran} \alpha=\left\langle a_{k}\right\rangle$. If $v \in V$, there exist scalars $y_{k}$ such that $v \beta=$ $\sum y_{k} a_{k}$ and so $v \beta=\left(\sum y_{k} u_{k}\right) \beta$. Hence, $v-\sum y_{k} u_{k} \in \operatorname{ker} \beta$ and thus $v \in \operatorname{ker} \beta \oplus$ $\left\langle u_{k}\right\rangle$. Therefore, $V=\operatorname{ker} \beta \oplus\left\langle u_{k}\right\rangle$ and, in this case, $n(\beta)=\operatorname{codim}\left\langle u_{k}\right\rangle=d(\lambda)+$ $\operatorname{dim}(\operatorname{ker} \alpha / \operatorname{ker} \lambda)$.

Lemma 8. If $\alpha, \beta \in A E(p, q)$ and $\alpha \mathcal{L} \beta$, then $\alpha \in A M(p, q)$ if and only if $\beta \in$ $A M(p, q)$.

Proof. This is identical to the proof of Lemma 5 using $\mathcal{L}$ in place of $\mathcal{R}$ and Theorem 4 in place of Theorem 2 .

Lemma 9. Let $\alpha \in A E(p, q)$ and denote the $\mathcal{L}$-class of $A E(p, q)$ containing $\alpha$ by $L_{\alpha}$. Then,

(a) $\alpha \in A M(p, q)$ implies $L_{\alpha}=\{\beta \in A E(p, q): \beta \in A M(p, q)$ and $\operatorname{ran} \beta=\operatorname{ran} \alpha\}$;

(b) $\alpha \notin A M(p, q)$ implies $L_{\alpha}=\{\beta \in A E(p, q): \operatorname{ran} \beta=\operatorname{ran} \alpha$ and $n(\beta)=n(\alpha)\}$.

Proof. Let $\beta \in A E(p, q)$ be such that $\alpha \mathcal{L} \beta$. Then, there exist $\lambda, \mu \in A E(p, q)$ such that $\alpha=\lambda \beta$ and $\beta=\mu \alpha$ (since $\operatorname{id}_{V} \in A E(p, q)$ ) and so $\operatorname{ran} \alpha=\operatorname{ran} \beta$. If $\alpha \in A M(p, q)$, then $\beta \in A M(p, q)$ (by Lemma 8). If $\alpha \notin A M(p, q)$, then $\beta \notin A M(p, q)$ (again, by Lemma 8) and so $n(\alpha) \geq q$ and $n(\beta) \geq q$. From Lemma 7 , we know that $n(\beta) \leq d(\lambda)+n(\alpha)$ and, similarly, $n(\alpha) \leq d(\mu)+n(\beta)$. Since $d(\lambda)<q \leq n(\alpha)$ and $d(\mu)<q \leq n(\beta)$, it follows that $d(\lambda)+n(\alpha)=n(\alpha)$ and $d(\mu)+n(\beta)=n(\beta)$. Hence, $n(\beta)=n(\alpha)$.

Conversely, suppose $\alpha \in A M(p, q), \beta \in A M(p, q) \cap A E(p, q)$ and $\operatorname{ran} \beta=\operatorname{ran} \alpha$. Then, as in the proof of Lemma 6, Hall's Theorem together with Lemma 2 imply that $\alpha \mathcal{L} \beta$ in $A M(p, q) \cap A E(p, q)$ and hence $\alpha \mathcal{L} \beta$ in $A E(p, q)$. That is, $\beta \in L_{\alpha}$ as required.

On the other hand, suppose $\alpha \notin A M(p, q), \beta \in A E(p, q), \operatorname{ran} \beta=\operatorname{ran} \alpha$ and $n(\beta)=$ $n(\alpha)$. Let $\operatorname{ran} \alpha=\left\langle e_{i}\right\rangle$, and choose $a_{i}, b_{i} \in V$ such that $a_{i} \alpha=e_{i}$ and $b_{i} \beta=e_{i}$ for each $i$. Clearly, $\left\{a_{i}\right\}$ is linearly independent. Moreover, if $\operatorname{ker} \alpha=\left\langle a_{k}\right\rangle$ then $V=\left\langle a_{i}\right\rangle \oplus\left\langle a_{k}\right\rangle$ : 
if $u \in V$ then $u \alpha=\sum x_{i} e_{i}=\left(\sum x_{i} a_{i}\right) \alpha$ for some scalars $x_{i}$, so $u-\sum x_{i} a_{i} \in \operatorname{ker} \alpha$; and clearly $\left\{a_{i}\right\} \cup\left\{a_{k}\right\}$ is linearly independent. Similarly, $V=\left\langle b_{i}\right\rangle \oplus\left\langle b_{k}\right\rangle$ where ker $\beta=\left\langle b_{k}\right\rangle$ and $|K|=n(\beta)=n(\alpha)$. Now write

$$
\left\{a_{k}\right\}=\left\{u_{k}\right\} \dot{\cup}\left\{u_{r}\right\}, \quad\left\{b_{k}\right\}=\left\{v_{k}\right\} \dot{\cup}\left\{v_{r}\right\},
$$

where $|R|<q$, and define $\lambda, \mu \in T(V)$ by

$$
\lambda=\left(\begin{array}{ccc}
a_{i} & u_{k} & u_{r} \\
b_{i} & v_{k} & 0
\end{array}\right), \quad \mu=\left(\begin{array}{ccc}
b_{i} & v_{k} & v_{r} \\
a_{i} & u_{k} & 0
\end{array}\right)
$$

Then $d(\lambda)=|R|<q$, so $\lambda \in A E(p, q)$ and likewise $\mu \in A E(p, q)$. Moreover, $\alpha=\lambda \beta$ and $\beta=\mu \alpha$, so $\alpha \mathcal{L} \beta$ in $A E(p, q)$ as required.

Next we describe the $\mathcal{D}$ and $\mathcal{J}$ relations on $A M(p, q)$, and the characterisation of its ideals follows from this.

Theorem 5. If $\alpha, \beta \in A M(p, q)$ then $\alpha \mathcal{D} \beta$ in $A M(p, q)$ if and only if one of the following occurs.

(a) $\alpha, \beta \in A E(p, q)$,

(b) $\alpha, \beta \notin A E(p, q)$ and $d(\alpha)=d(\beta)$.

Proof. Suppose $\alpha \mathcal{L} \gamma \mathcal{R} \beta$ in $A M(p, q)$. If $\beta \in A E(p, q)$ then $\gamma \in A E(p, q)$ (by Lemma 5): that is, $d(\gamma)<q$ and, since $\operatorname{ran} \alpha=\operatorname{ran} \gamma$, this implies $d(\alpha)<q$. Hence $\alpha \in A E(p, q)$. On the other hand, if $\beta \notin A E(p, q)$ then, by Lemma $6(\mathrm{~b}), d(\alpha)=d(\gamma)=$ $d(\beta) \geq q$ and hence $\alpha \notin A E(p, q)$. For the converse, we start by writing

$$
\alpha=\left(\begin{array}{cc}
e_{j} & e_{i} \\
0 & a_{i}
\end{array}\right), \quad \beta=\left(\begin{array}{cc}
f_{k} & f_{i} \\
0 & b_{i}
\end{array}\right)
$$

(this is possible since $\alpha, \beta \in A M(p, q)$ implies $r(\alpha)=r(\beta)=p)$. Now define $\gamma \in T(V)$ by

$$
\gamma=\left(\begin{array}{cc}
f_{k} & f_{i} \\
0 & a_{i}
\end{array}\right)
$$

If $\alpha, \beta \in A E(p, q)$, then $n(\gamma)=n(\beta)<q$ and $d(\gamma)=d(\alpha)<q$, so $\gamma \in A M(p, q) \cap$ $A E(p, q)$. In fact, $\operatorname{ran} \gamma=\operatorname{ran} \alpha$ and $\operatorname{ker} \gamma=\operatorname{ker} \beta$, so $\alpha \mathcal{L} \gamma$ and $\gamma \mathcal{R} \beta$, and hence $\alpha \mathcal{D} \beta$ in $A M(p, q)$. However, if $\alpha, \beta \notin A E(p, q)$ and $d(\alpha)=d(\beta)$, then $\gamma \in A M(p, q)$ (as before) and $\operatorname{ran} \gamma=\operatorname{ran} \alpha$, so $\alpha \mathcal{L} \gamma$ by Lemma 2. Also, $\operatorname{ker} \gamma=\operatorname{ker} \beta$ and $d(\gamma)=$ $d(\alpha)=d(\beta)$, so $\gamma \mathcal{R} \beta$ by Lemma $6(\mathrm{~b})$. In other words, we have shown that $\alpha \mathcal{D} \beta$ in $A M(p, q)$.

Corollary 1. $\mathcal{D}=\mathcal{J}$ on $A M(p, q)$.

Proof. We know $\mathcal{D} \subseteq \mathcal{J}$. Therefore, since $\mathcal{D}$ is universal on $A M(p, q) \cap A E(p, q)$ by Theorem 5(a), $\mathcal{J}$ is also. Now suppose $\alpha=\lambda \beta \mu$ and $\beta=\lambda^{\prime} \alpha \mu^{\prime}$ for some $\lambda, \mu, \lambda^{\prime}, \mu^{\prime} \in$ $A M(p, q)$. By Lemma 4, we have

$$
d(\beta) \leq d(\lambda \beta) \leq n(\mu)+\operatorname{dim}(\operatorname{ran} \mu / \operatorname{ran} \alpha) \leq n(\mu)+d(\alpha) .
$$

Hence if $\beta \notin A E(p, q)$ then $q \leq d(\beta) \leq n(\mu)+d(\alpha)$, and $n(\mu)<q$, so $d(\alpha) \geq q$ and thus $\alpha \notin A E(p, q)$. Likewise, using $\beta=\lambda^{\prime} \alpha \mu^{\prime}$, we find that $\alpha \notin A E(p, q)$ implies 
$\beta \notin A E(p, q)$. That is, if $\alpha \mathcal{J} \beta$ in $A M(p, q)$ then either $\alpha, \beta \in A M(p, q) \cap A E(p, q)$ or $\alpha, \beta \notin A E(p, q)$. In the latter case, we have $d(\beta) \leq n(\mu)+d(\alpha)=d(\alpha)$ since $n(\mu)<q \leq d(\alpha)$. Similarly, $\beta=\lambda^{\prime} \alpha \mu^{\prime}$ implies $d(\alpha) \leq d(\beta)$, and equality follows. Thus, $\alpha \mathcal{D} \beta$ by Theorem 5(b). Hence, in both cases, $\alpha \mathcal{J} \beta$ implies $\alpha \mathcal{D} \beta$.

Theorem 6. The proper ideals of $A M(p, q)$ are precisely the sets

$$
M_{\xi}=\{\alpha \in A M(p, q): d(\alpha) \geq \xi\},
$$

where $q \leq \xi \leq p$. In fact, each $M_{\xi}$ is a principal ideal of $A M(p, q)$ generated by an element with defect $\xi$.

Proof. Let $\xi$ be a cardinal such that $q \leq \xi \leq p$. By Lemma 4 , given $\alpha \in M_{\xi}$ and $\lambda, \mu \in A M(p, q)$, we have

$$
\xi \leq d(\alpha) \leq d(\lambda \alpha) \leq n(\mu)+\operatorname{dim}(\operatorname{ran} \mu / \operatorname{ran}(\lambda \alpha \mu)) \leq n(\mu)+d(\lambda \alpha \mu) .
$$

Since $n(\mu)<q$ and $\xi \geq q$, we see that $d(\lambda \alpha \mu) \geq \xi$. Therefore, $\lambda \alpha \mu \in M_{\xi}$ and so $M_{\xi}$ is an ideal of $A M(p, q)$ (note that $\lambda$ and $\mu$ can equal $\operatorname{id}_{V} \in A M(p, q)$ ).

Conversely, let $I$ be an ideal of $A M(p, q)$. If there exists $\alpha \in I \cap A E(p, q)$ then $\alpha \in A M(p, q) \cap A E(p, q)$ and, since $\operatorname{id}_{V} \in A M(p, q) \cap A E(p, q)$, Theorem 5(a) implies $\operatorname{id}_{V} \mathcal{D} \alpha$. Consequently, by Corollary 1, we have $\operatorname{id}_{V} \in J(\alpha)$, the principal ideal of $A M(p, q)$ generated by $\alpha$, so $\operatorname{id}_{V} \in I$ and hence $I=A M(p, q)$. Now suppose $I \cap A E(p, q)=\emptyset$ and choose $\gamma \in I$ with minimal defect $\xi$. Note that $d(\beta) \geq d(\gamma)=\xi$ for every $\beta \in I$ and, clearly, $q \leq \xi \leq p$. Hence,

$$
A M(p, q) \gamma A M(p, q) \subseteq I \subseteq M_{\xi}
$$

Given $\alpha \in M_{\xi}$, we have $d(\alpha) \geq \xi=d(\gamma)$. In the usual way, write

$$
\alpha=\left(\begin{array}{cc}
e_{j} & e_{i} \\
0 & a_{i}
\end{array}\right), \quad \gamma=\left(\begin{array}{cc}
f_{k} & f_{i} \\
0 & b_{i}
\end{array}\right)
$$

(note that this is possible since $\alpha, \gamma \in A M(p, q)$ implies $r(\alpha)=r(\gamma)=p$ ). Since $\left\{b_{i}\right\}$ is a basis for ran $\gamma$, it can be expanded to a basis for $V$, say $\left\{b_{i}\right\} \dot{\cup}\left\{b_{\ell}\right\}$, with $|L|=d(\gamma)=\xi$. Similarly, $\left\{a_{i}\right\}$ is a basis for $\operatorname{ran} \alpha$ and it can be expanded to a basis $\left\{a_{i}\right\} \dot{\cup}\left\{a_{r}\right\} \dot{\cup}\left\{a_{\ell}\right\}$ for $V$, where $|R|+|L|=d(\alpha)$ (note that $d(\alpha) \geq d(\gamma)=|L|$ ). Now define $\lambda, \mu \in T(V)$ by

$$
\lambda=\left(\begin{array}{cc}
e_{j} & e_{i} \\
0 & f_{i}
\end{array}\right), \quad \mu=\left(\begin{array}{cc}
b_{i} & b_{\ell} \\
a_{i} & a_{\ell}
\end{array}\right)
$$

Clearly, $n(\lambda)=n(\alpha)<q$ and $n(\mu)=0$, and hence $\lambda, \mu \in A M(p, q)$. Also $\alpha=\lambda \gamma \mu$ and, since $I$ is an ideal, $\gamma \in I$ implies $\alpha \in I$. Therefore, $I=M_{\xi}$ and, in effect, we have shown that $I$ is a principal ideal generated by an element with defect $\xi$.

Clearly, the proper ideals of $A M(p, q)$ form a chain under $\subseteq$, with the smallest being $M_{p}$ and the largest being $M_{q}$.

Now we proceed to characterise the $\mathcal{D}$ and $\mathcal{J}$ relations on $A E(p, q)$ and, using this, we describe the ideal structure of $A E(p, q)$. 
Theorem 7. If $\alpha, \beta \in A E(p, q)$ then $\alpha \mathcal{D} \beta$ in $A E(p, q)$ if and only if one of the following occurs.

(a) $\alpha, \beta \in A M(p, q)$,

(b) $\alpha, \beta \notin A M(p, q)$ and $n(\alpha)=n(\beta)$.

Proof. Suppose $\alpha \mathcal{L} \gamma \mathcal{R} \beta$ in $A E(p, q)$. If $\alpha \in A M(p, q)$ then $\gamma \in A M(p, q)$ (by Lemma 8) and hence $n(\gamma)<q$. Since $\operatorname{ker} \gamma=\operatorname{ker} \beta$, we have $n(\beta)=n(\gamma)<q$ and so $\beta \in A M(p, q)$. Conversely, if $\alpha, \beta \in A M(p, q) \cap A E(p, q)$ then the same argument as that used in the proof of Theorem 5 (a) shows that $\alpha \mathcal{D} \beta$ in $A E(p, q)$.

Now assume $\alpha \mathcal{L} \gamma \mathcal{R} \beta$ in $A E(p, q)$ and $\alpha \notin A M(p, q)$. Then, $\operatorname{ker} \beta=\operatorname{ker} \gamma$ and so, by Lemma $9(\mathrm{~b}), n(\beta)=n(\gamma)=n(\alpha) \geq q$, so $\beta \notin A M(p, q)$. Conversely, suppose $\alpha, \beta \notin A M(p, q)$ and $n(\alpha)=n(\beta)$ and, in the usual way, write

$$
\alpha=\left(\begin{array}{cc}
e_{j} & e_{i} \\
0 & a_{i}
\end{array}\right), \quad \beta=\left(\begin{array}{cc}
f_{j} & f_{i} \\
0 & b_{i}
\end{array}\right)
$$

(note that this is possible since $d(\alpha)<q$ and $d(\beta)<q$ imply $r(\alpha)=r(\beta)=p$ ). Now define $\gamma \in T(V)$ by

$$
\gamma=\left(\begin{array}{cc}
f_{j} & f_{i} \\
0 & a_{i}
\end{array}\right)
$$

Then, $d(\gamma)=d(\alpha)<q$, so $\gamma \in A E(p, q)$. In fact, $\operatorname{ker} \gamma=\operatorname{ker} \beta$ and so $\gamma \mathcal{R} \beta$. Also, $\operatorname{ran} \gamma=\operatorname{ran} \alpha$ and $n(\gamma)=n(\beta)=n(\alpha)$. Hence $\alpha \mathcal{L} \gamma$. In other words, we have shown $\alpha \mathcal{D} \beta$ in $A E(p, q)$.

Corollary 2. $\mathcal{D}=\mathcal{J}$ on $A E(p, q)$.

Proof. Since $\mathcal{D} \subseteq \mathcal{J}$ and $\mathcal{D}$ is universal on $A M(p, q) \cap A E(p, q)$, so is $\mathcal{J}$. Now suppose $\alpha=\lambda \beta \mu$ and $\beta=\lambda^{\prime} \alpha \mu^{\prime}$ for some $\lambda, \mu, \lambda^{\prime}, \mu^{\prime} \in A E(p, q)$. By Lemma 7 , it follows that

$$
n(\beta) \leq n(\beta \mu) \leq d(\lambda)+\operatorname{dim}(\operatorname{ker} \alpha / \operatorname{ker} \lambda) \leq d(\lambda)+n(\alpha) .
$$

Therefore, if $\beta \notin A M(p, q)$ then $q \leq n(\beta) \leq d(\lambda)+n(\alpha)$, and $d(\lambda)<q$, so $n(\alpha) \geq q$. Hence $\alpha \notin A M(p, q)$. Likewise, using $\beta=\lambda^{\prime} \alpha \mu^{\prime}$, we conclude that $\alpha \notin A M(p, q)$ implies $\beta \notin A M(p, q)$. Thus, if $\alpha \mathcal{J} \beta$ in $A E(p, q)$, then $\alpha \in A M(p, q)$ if and only if $\beta \in A M(p, q)$. Moreover, if $\alpha, \beta \notin A M(p, q)$ then $n(\beta) \leq d(\lambda)+n(\alpha)=n(\alpha)$ and $n(\alpha) \leq d\left(\lambda^{\prime}\right)+n(\beta)=n(\beta)$. Hence $n(\alpha)=n(\beta)$ and so $\alpha \mathcal{D} \beta$ by Theorem $7(\mathrm{~b})$. Thus we have shown that $\mathcal{J} \subseteq \mathcal{D}$ on $A E(p, q)$.

Theorem 8. The proper ideals of $A E(p, q)$ are precisely the sets

$$
E_{\xi}=\{\alpha \in A E(p, q): n(\alpha) \geq \xi\}
$$

where $q \leq \xi \leq p$. In fact, each $E_{\xi}$ is a principal ideal of $A E(p, q)$ generated by an element with nullity $\xi$.

Proof. Let $\xi$ be an infinite cardinal such that $q \leq \xi \leq p$, and suppose $\alpha \in E_{\xi}$ and $\lambda, \mu \in A E(p, q)$. By Lemma 7 , we have

$$
\xi \leq n(\alpha) \leq n(\alpha \mu) \leq d(\lambda)+\operatorname{dim}(\operatorname{ker}(\lambda \alpha \mu) / \operatorname{ker} \lambda) \leq d(\lambda)+n(\lambda \alpha \mu) .
$$


Since $\lambda \in A E(p, q)$, we know $d(\lambda)<q$, and $q \leq \xi$ by supposition. Hence $n(\lambda \alpha \mu) \geq \xi$ and so $\lambda \alpha \mu \in E_{\xi}$. Therefore, $E_{\xi}$ is an ideal of $A E(p, q)$, since $\operatorname{id}_{V} \in A E(p, q)$.

Conversely, let $I$ be an ideal of $A E(p, q)$. If there exists $\alpha \in I \cap A M(p, q)$ then $\alpha \in A E(p, q) \cap A M(p, q)$ and, since $\mathrm{id}_{V} \in A E(p, q) \cap A M(p, q)$, Theorem 7(a) implies $\operatorname{id}_{V} \mathcal{D} \alpha$. Consequently, by Corollary 2, we have $\operatorname{id}_{V} \in J(\alpha)$, the principal ideal of $A E(p, q)$ generated by $\alpha$, so $\operatorname{id}_{V} \in I$ and hence $I=A E(p, q)$. Finally, suppose $I \cap A M(p, q)=\emptyset$ and choose $\epsilon \in I$ with minimal nullity $\xi$. Then, $q \leq \xi \leq p$ and $n(\beta) \geq n(\epsilon) \geq \xi$ for every $\beta \in I$. Therefore,

$$
A E(p, q) \epsilon A E(p, q) \subseteq I \subseteq E_{\xi}
$$

Let $\alpha \in E_{\xi}$. Then $n(\alpha) \geq \xi=n(\epsilon)$. Now let $\left\{f_{k}\right\}$ be a basis for ker $\epsilon$, with $|K|=\xi$, and expand it to a basis for $V$, say $\left\{f_{k}\right\} \cup \dot{\cup}\left\{f_{i}\right\}$. For every $i$, write $f_{i} \epsilon=b_{i}$. Clearly, $\left\{b_{i}\right\}$ is a basis for $\operatorname{ran} \epsilon$, and $\epsilon \in A E(p, q)$ implies $|I|=r(\epsilon)=p$. Likewise, let $\left\{e_{j}\right\} \cup \dot{\cup}\left\{e_{k}\right\}$ be a basis for $\operatorname{ker} \alpha$, with $|J|+|K|=n(\alpha) \geq n(\epsilon)=|K|$, and expand it to a basis $\left\{e_{j}\right\} \cup \dot{\cup}\left\{e_{k}\right\} \cup \dot{\cup}\left\{e_{r}\right\}$ for $V$. For each $r$, write $e_{r} \alpha=a_{r}$. Since $\alpha \in A E(p, q)$ and $\left\{a_{r}\right\}$ is a basis for $\operatorname{ran} \alpha$, we know $r(\alpha)=p$, and hence we can write $\left\{e_{i}\right\}$ and $\left\{a_{i}\right\}$ instead of $\left\{e_{r}\right\}$ and $\left\{a_{r}\right\}$, respectively. Expand $\left\{b_{i}\right\}$ to a basis for $V$, say $\left\{b_{i}\right\} \dot{\cup}\left\{b_{\ell}\right\}$, and define $\lambda, \mu \in T(V)$ by

$$
\lambda=\left(\begin{array}{ccc}
e_{j} & e_{k} & e_{i} \\
0 & f_{k} & f_{i}
\end{array}\right), \quad \mu=\left(\begin{array}{cc}
b_{i} & b_{\ell} \\
a_{i} & 0
\end{array}\right) .
$$

Clearly, $d(\lambda)=0$ and $d(\mu)=d(\alpha)<q$, and hence $\lambda, \mu \in A E(p, q)$. Also, $\alpha=\lambda \epsilon \mu$ and so $\alpha \in I$, since $I$ is an ideal of $A E(p, q)$ and $\epsilon \in I$. Therefore, $I=E_{\xi}$ and, in effect, we have shown that $I$ is a principal ideal generated by an element with nullity $\xi$.

It is now easy to see that the proper ideals of $A E(p, q)$ form a chain under $\subseteq$, with the smallest being $E_{p}$ and the largest being $E_{q}$.

\section{Maximal right simple subsemigroups}

In [7] Theorem 7, the author proved that if $q \leq \xi \leq p$, then the linear Baer-Levi semigroups

$$
G S(p, \xi)=\{\alpha \in T(V): n(\alpha)=0, d(\alpha)=\xi\},
$$

are precisely the maximal right simple subsemigroups of $K N(p, q)=\{\alpha \in T(V)$ : $n(\alpha)=0, d(\alpha) \geq q\}$ when $q<p$. It is not difficult to show that each $G S(p, \xi)$ is a maximal right simple subsemigroup of $A M(p, q)$ (even if $p=q$ ). In fact, we will determine all maximal right simple subsemigroups of $A M(p, q)$. To do this, we need two preliminary results.

Lemma 10. For each infinite cardinal $\xi$ such that $\xi \leq p$, and for each subspace $A$ of $V$ with $\operatorname{dim} A<q$, the set

$$
M(A, \xi)=\{\alpha \in T(V): \operatorname{ker} \alpha=A, \operatorname{ran} \alpha \cap A=\{0\}, \operatorname{dim} V /(\operatorname{ran} \alpha \oplus A)=\xi\}
$$

is a maximal right simple subsemigroup of $A M(p, q)$.

Proof. Clearly, $M(A, \xi) \subseteq A M(p, q)$ and it is non-empty. For example, if $V=\left\langle a_{j}\right\rangle \oplus$ $\left\langle a_{i}\right\rangle$ where $A=\left\langle a_{j}\right\rangle$ and $|I|=p$ (possible since $\operatorname{dim} A<q \leq p$ ), we can write 
$\left\{a_{i}\right\}=\left\{b_{i}\right\} \dot{\cup}\left\{b_{k}\right\}$ where $|K|=\xi$ and define $\pi \in M(A, \xi)$ by

$$
\pi=\left(\begin{array}{cc}
a_{j} & a_{i} \\
0 & b_{i}
\end{array}\right) .
$$

Let $\alpha, \beta \in M(A, \xi)$. Then, $(\operatorname{ker}(\alpha \beta)) \alpha=\operatorname{ran} \alpha \cap \operatorname{ker} \beta=\{0\}$ and so $\operatorname{ker}(\alpha \beta) \subseteq \operatorname{ker} \alpha$. Since $\operatorname{ker} \alpha \subseteq \operatorname{ker}(\alpha \beta)$ always, it follows that $\operatorname{ker}(\alpha \beta)=A$. Also $\operatorname{ran}(\alpha \beta) \subseteq \operatorname{ran} \beta$ implies $\operatorname{ran}(\alpha \beta) \cap A \subseteq\{0\}$, and equality follows. Now suppose $\left\{a_{j}\right\}$ is a basis for $A$ and expand it to a basis $\left\{a_{j}\right\} \dot{\cup}\left\{a_{i}\right\}$ for $V$, with $|I|=\operatorname{codim} A=p$. For each $i$, write $a_{i} \alpha=e_{i}$. Then $\left\{e_{i}\right\}$ is a basis for ran $\alpha$, and $\operatorname{ran} \alpha \cap A=\{0\}$ implies $V=\left\langle a_{j}\right\rangle \oplus\left\langle e_{i}\right\rangle \oplus\left\langle e_{k}\right\rangle$ for some linearly independent $\left\{e_{k}\right\} \subseteq V$, where $|K|=\operatorname{dim} V /(\operatorname{ran} \alpha \oplus A)=\xi$. Now write $e_{i} \beta=f_{i}$ and $e_{k} \beta=f_{k}$ for every $i$ and every $k$, respectively. Since ker $\beta=A$, we know that $\left\{f_{i}\right\} \dot{\cup}\left\{f_{k}\right\}$ is a basis for $\operatorname{ran} \beta$, and hence it can be expanded to a basis for $V$, say $\left\{f_{i}\right\} \dot{\cup}\left\{f_{k}\right\} \cup \dot{\cup}\left\{c_{k}\right\} \dot{\cup}\left\{a_{j}\right\}$ (recall that $\operatorname{ran} \beta \cap A=\{0\}$ and $\operatorname{dim} V /(\operatorname{ran} \beta \oplus A)=$ $\xi=|K|)$. Clearly, we have

$$
\alpha \beta=\left(\begin{array}{cc}
a_{j} & a_{i} \\
0 & f_{i}
\end{array}\right)
$$

Hence $\operatorname{dim} V /(\operatorname{ran}(\alpha \beta) \oplus A)=\operatorname{dim}\left\langle f_{k}, c_{k}\right\rangle=\xi+\xi=\xi$ (since $\xi$ is infinite). Therefore, $\alpha \beta \in M(A, \xi)$ and so $M(A, \xi)$ is a subsemigroup of $A M(p, q)$.

Next we show that $M(A, \xi)$ is right simple. To do this, write $a_{i} \beta=c_{i}$ for every $i$. Since $\operatorname{ker} \beta=A$, we know $\left\{c_{i}\right\}$ is a basis for $\operatorname{ran} \beta$, and hence it can be expanded to a basis for $V$, say $\left\{c_{i}\right\} \dot{\cup}\left\{g_{k}\right\} \dot{\cup}\left\{a_{j}\right\}$ (note that $\operatorname{ran} \beta \cap A=\{0\}$ and $\operatorname{dim} V /(\operatorname{ran} \beta \oplus A)=\xi=|K|$ ). Now write $\left\{g_{k}\right\}=\left\{u_{k}\right\} \cup \dot{\cup}\left\{v_{k}\right\}$ (possible since $|K|=\xi \geq \aleph_{0}$ ) and define $\lambda$ in $T(V)$ by

$$
\lambda=\left(\begin{array}{ccc}
a_{j} & e_{i} & e_{k} \\
0 & c_{i} & u_{k}
\end{array}\right) .
$$

Then, $\operatorname{ker} \lambda=A, \operatorname{ran} \lambda \cap A=\{0\}$ and $\operatorname{dim} V /(\operatorname{ran} \lambda \oplus A)=\xi$, so $\lambda \in M(A, \xi)$. Also $\beta=\alpha \lambda$, and we have shown $M(A, \xi)$ is right simple.

Next suppose $M(A, \xi) \subseteq M \subseteq A M(p, q)$ where $M$ is a right simple subsemigroup of $A M(p, q)$. Since $A M(p, q)$ is not right simple (see the remark before Theorem 2), it follows that $M \neq A M(p, q)$. Let $\alpha \in M$ and $\gamma \in M(A, \xi)$. If $\alpha=\gamma$ then $\alpha \in M(A, \xi)$. Suppose $\alpha \neq \gamma$. Both $\alpha$ and $\gamma$ are elements of $M$ and, since this semigroup is right simple, there exist $\lambda, \mu \in M$ such that $\alpha=\gamma \lambda$ and $\gamma=\alpha \mu$ : that is, $\alpha \mathcal{R} \gamma$ in $M$, and hence in $A M(p, q)$ also. By Lemma 6 we have $\operatorname{ker} \alpha=\operatorname{ker} \gamma=A$. Now suppose there exists a non-zero $v=u \alpha \in \operatorname{ran} \alpha \cap A$. Then $u \notin A=\operatorname{ker} \alpha$ and so $\operatorname{ker} \gamma \varsubsetneqq A \oplus\langle u\rangle \subseteq$ $\operatorname{ker}(\alpha \gamma)$. From Lemma 6, we deduce that $\gamma$ and $\alpha \gamma$ are not $\mathcal{R}$-related in $A M(p, q)$, and hence $\alpha \gamma \notin M$ since $M$ is right simple. But this contradicts the fact that $M$ is closed, so $\operatorname{ran} \alpha \cap A=\{0\}$. Next, we claim that $\operatorname{dim} V /(\operatorname{ran} \alpha \oplus A)=\operatorname{dim} V /(\operatorname{ran} \gamma \oplus A)$.

First, since $\lambda, \mu \in M$, an argument similar to the one above shows that ker $\lambda=A=$ ker $\mu$ and $\operatorname{ran} \lambda \cap A=\{0\}=\operatorname{ran} \mu \cap A$. Next, we adopt the same notation as in the second paragraph of this proof, albeit for a different $\alpha$. Now write $a_{i} \gamma=g_{i}$ for each $i$. Then $\left\{g_{i}\right\}$ is a basis for $\operatorname{ran} \gamma$ (since $\operatorname{ker} \gamma=A=\left\langle a_{j}\right\rangle$ ) and it can be expanded to a basis for $V$, say $\left\{g_{i}\right\} \dot{\cup}\left\{a_{j}\right\} \dot{\cup}\left\{g_{\ell}\right\}$, where $|L|=\operatorname{dim} V /(\operatorname{ran} \gamma \oplus A)=\xi$ since $\gamma \in M(A, \xi)$. Clearly, $e_{i}=a_{i} \alpha=g_{i} \lambda$ for each $i$ and, since ker $\lambda=A$, we deduce that $\operatorname{ran} \lambda=\left\langle e_{i}\right\rangle \oplus\left\langle g_{\ell} \lambda\right\rangle$. Consequently, since $\operatorname{ran} \alpha=\left\langle e_{i}\right\rangle$ and $\operatorname{ran} \lambda \cap A=\{0\}$, we obtain

$$
\operatorname{dim} V /(\operatorname{ran} \alpha \oplus A)=\operatorname{codim}\left\langle e_{i}, a_{j}\right\rangle=|K| \geq|L| .
$$


Likewise, $\gamma=\alpha \mu$ implies $|K| \leq|L|$. Thus, our claim is valid. Hence $\alpha$ belongs to $M(A, \xi)$, and so $M(A, \xi)=M$. Therefore, $M(A, \xi)$ is a maximal right simple subsemigroup of $A M(p, q)$.

Note that for each cardinal $\xi$ such that $q \leq \xi \leq p$, we have $G S(p, \xi)=M(\{0\}, \xi)$, and hence each $G S(p, \xi)$ is a maximal right simple subsemigroup of $A M(p, q)$, as observed before.

Clearly, the general linear group $G(V)$ is a right simple subsemigroup of $A M(p, q)$. In fact, it is maximal under these conditions. For, suppose $G(V) \subseteq M \subseteq A M(p, q)$ for some right simple subsemigroup $M$ of $A M(p, q)$. Then, given $\alpha \in M$ and $\gamma \in G(V)$, we have $\alpha \mathcal{R} \gamma$ in $M$ and hence also in $A M(p, q)$, so ker $\alpha=\operatorname{ker} \gamma=\{0\}$ by Lemma 6 . In fact, if $\alpha=\gamma \lambda$ and $\gamma=\alpha \mu$ for some $\lambda, \mu \in M$ then, since $M$ is right simple, $\lambda$ and $\mu$ are $\mathcal{R}$-related to $\gamma \in M$ and so ker $\lambda=\{0\}=\operatorname{ker} \mu$ as before. Therefore, by Lemma 4 ,

$$
d(\gamma) \leq n(\lambda)+d(\alpha)=d(\alpha) \leq n(\mu)+d(\gamma)=d(\gamma)
$$

Hence, $d(\alpha)=0=n(\alpha)$ and $\alpha \in G(V)$. In fact, the next result gives a class of maximal right simple subsemigroups of $A M(p, q)$ which contains $G(V)$ (with a slight abuse of terminology, we observe that $G(V)=N(B, \zeta)$ precisely when $\zeta=0$ and $B=\{0\}$ ).

Lemma 11. For every cardinal $\zeta<q$ and every subspace $B$ of $V$ with dimension $\zeta$, the set

$$
N(B, \zeta)=\{\alpha \in T(V): \operatorname{ker} \alpha=B, V=\operatorname{ran} \alpha \oplus B\}
$$

is a maximal right simple subsemigroup of $A M(p, q)$.

Proof. Clearly, $N(B, \zeta) \subseteq A M(p, q)$ and it is non-empty. For example, if $V=\left\langle b_{j}\right\rangle \oplus\left\langle b_{i}\right\rangle$ where $B=\left\langle b_{j}\right\rangle,|J|=\zeta$ and $|I|=\operatorname{codim} B$, we can define $\alpha \in N(B, \zeta)$ by

$$
\alpha=\left(\begin{array}{cc}
b_{j} & b_{i} \\
0 & b_{i}
\end{array}\right)
$$

Let $\alpha, \beta \in N(B, \zeta)$. Then, $\operatorname{ran} \alpha \cap B=\{0\}$ implies $(\operatorname{ker}(\alpha \beta)) \alpha=\{0\}$, and hence $\operatorname{ker}(\alpha \beta) \subseteq B$. Since $B=\operatorname{ker} \alpha \subseteq \operatorname{ker}(\alpha \beta)$, we have $\operatorname{ker}(\alpha \beta)=B$. Clearly $\operatorname{ran}(\alpha \beta) \subseteq$ $\operatorname{ran} \beta$. Now, if $v \in V$, then $v=a+b$ for some $a \in \operatorname{ker} \alpha=\operatorname{ker} \beta$ and $b \in \operatorname{ran} \alpha$. Therefore, there exists $u \in V$ such that $b=u \alpha$ and $v \beta=a \beta+b \beta=u(\alpha \beta)$. Hence, $\operatorname{ran}(\alpha \beta)=\operatorname{ran} \beta$ and so $\alpha \beta \in N(B, \zeta)$.

Now suppose $\left\{b_{j}\right\}$ is a basis for $B$ and expand it to a basis $\left\{b_{j}\right\} \dot{\cup}\left\{b_{i}\right\}$ for $V$. For each $i$, write $b_{i} \alpha=e_{i}$ and $b_{i} \beta=f_{i}$. Since $\left\{e_{i}\right\}$ and $\left\{f_{i}\right\}$ are bases for $\operatorname{ran} \alpha$ and $\operatorname{ran} \beta$, respectively, we have $V=\left\langle e_{i}\right\rangle \oplus\left\langle b_{j}\right\rangle=\left\langle f_{i}\right\rangle \oplus\left\langle b_{j}\right\rangle$. Define $\lambda \in T(V)$ by

$$
\lambda=\left(\begin{array}{cc}
e_{i} & b_{j} \\
f_{i} & 0
\end{array}\right) .
$$

Clearly, $\lambda \in N(B, \zeta)$ and $\beta=\alpha \lambda$. In other words, $N(B, \zeta)$ is right simple.

We have just proved that $N(B, \zeta)$ is a right simple subsemigroup of $A M(p, q)$ : next we show it is maximal under these conditions. To do this, suppose $N(B, \zeta) \subseteq M \subseteq$ $A M(p, q)$, where $M$ is a right simple subsemigroup of $A M(p, q)$. As before, $M \neq$ $A M(p, q)$ since the latter is not right simple. Now let $\alpha \in M$ and $\gamma \in N(B, \zeta)$. If 
$\alpha=\gamma$ then $\alpha \in N(B, \zeta)$. Now suppose $\alpha \neq \gamma$. Clearly, $\alpha, \gamma \in M$ and so $\alpha=\gamma \lambda$ and $\gamma=\alpha \mu$ for some $\lambda, \mu \in M$. Since $d(\gamma)=\zeta<q$, we have $\gamma \in A E(p, q)$, and hence Lemma 6(a) implies $d(\alpha)<q$ and $\operatorname{ker} \alpha=\operatorname{ker} \gamma=B$. As in the proof of Lemma 10, if $\operatorname{ran} \alpha \cap B \neq\{0\}$ then $\gamma$ and $\alpha \gamma$ are not $\mathcal{R}$-related in $A M(p, q)$, which implies $\alpha \gamma \notin M$, a contradiction. Therefore $\operatorname{ran} \alpha \cap B=\{0\}$. Likewise, by considering $\lambda, \gamma \in M$ and $\mu, \gamma \in M$, we deduce that $\operatorname{ker} \lambda=B=\operatorname{ker} \mu$ and $\operatorname{ran} \lambda \cap B=\{0\}=\operatorname{ran} \mu \cap B$. Suppose ran $\alpha \oplus B \varsubsetneqq V$ and write $V=\left\langle e_{i}\right\rangle \oplus\left\langle b_{j}\right\rangle \oplus\left\langle v_{s}\right\rangle$, where $\left\{b_{j}\right\}$ is a basis for $B,\left\{b_{j}\right\} \dot{\cup}\left\{b_{i}\right\}$ is a basis for $V$ and $e_{i}=b_{i} \alpha$ for each $i$. Since $b_{i} \gamma=\left(b_{i} \alpha\right) \mu=e_{i} \mu$ and $V=\left\langle b_{i} \gamma\right\rangle \oplus\left\langle b_{j}\right\rangle$, we have $V=\left\langle e_{i} \mu\right\rangle \oplus\left\langle b_{j}\right\rangle \varsubsetneqq\left\langle e_{i} \mu\right\rangle \oplus\left\langle v_{s} \mu\right\rangle \oplus\left\langle b_{j}\right\rangle \subseteq V$, a contradiction since $S \neq \emptyset$ and $\left\langle v_{s}\right\rangle \cap \operatorname{ker} \mu=\{0\}$. Hence ran $\alpha \oplus B=V$. Thus, $\alpha \in N(B, \zeta)$ and $M=N(B, \zeta)$. Therefore, $N(B, \zeta)$ is a maximal right simple subsemigroup of $A M(p, q)$.

Theorem 9. The maximal right simple subsemigroups of $A M(p, q)$ are exactly the sets $M(A, \xi)$, where $A$ is a subspace of $V$ with $\operatorname{dim} A<q$ and $\xi$ is an infinite cardinal such that $\xi \leq p$, and the sets $N(B, \zeta)$, where $\zeta$ is a cardinal such that $\zeta<q$ and $B$ is a subspace of $V$ with $\operatorname{dim} B=\zeta$.

Proof. By Lemma 10, each $M(A, \xi)$ is a maximal right simple subsemigroup of $A M(p, q)$; and by Lemma 11 , so is each $N(B, \zeta)$. Now suppose $M$ is a maximal right simple subsemigroup of $A M(p, q)$ and let $\alpha \in M$. For every $\beta \in M, \alpha$ and $\beta$ are $\mathcal{R}$ related in $A M(p, q)$, and hence $\operatorname{ker} \alpha=\operatorname{ker} \beta$. Let $A=\operatorname{ker} \alpha$. As in the proof of Lemma 10 , if $\operatorname{ran} \beta \cap A \neq\{0\}$ for some $\beta \in M$, then $A \varsubsetneqq \operatorname{ker}(\beta \alpha)$ and so $\beta \alpha \notin M$, a contradiction. Therefore, $\operatorname{ran} \beta \cap A=\{0\}$ for every $\beta \in M$ : in particular, we have $d(\beta) \geq \operatorname{dim} A$. Suppose $\beta \neq \alpha$. Since $M$ is right simple, there exist $\lambda, \mu \in M$ such that $\alpha=\beta \lambda$ and $\beta=\alpha \mu$. Since $\lambda, \mu \in M$, we have ker $\lambda=A=\operatorname{ker} \mu$ and $\operatorname{ran} \lambda \cap A=\{0\}=\operatorname{ran} \mu \cap A$. In fact, using an argument similar to that in the proof of Lemma 10, we can show that $\operatorname{dim} V /(\operatorname{ran} \alpha \oplus A)=\operatorname{dim} V /(\operatorname{ran} \beta \oplus A)$. Let $\xi=\operatorname{dim} V /(\operatorname{ran} \alpha \oplus A)$ and suppose $\xi \geq \aleph_{0}$. Then, $M \subseteq M(A, \xi)$ and, by the maximality of $M$, it follows that $M=M(A, \xi)$.

On the other hand, if $\xi$ is finite then it must be 0 : that is, we claim that in this case $V=\operatorname{ran} \beta \oplus A$ for every $\beta \in M$. For, suppose $\operatorname{ran} \alpha \oplus A \varsubsetneqq V$ and write, in the usual way,

$$
\alpha=\left(\begin{array}{cc}
a_{j} & a_{i} \\
0 & e_{i}
\end{array}\right)
$$

Now expand $\left\{e_{i}\right\}$ to a basis $\left\{e_{i}\right\} \dot{\cup}\left\{a_{j}\right\} \dot{\cup}\left\{e_{k}\right\}$ for $V$, with $|K|=\xi<\aleph_{0}$. Write $e_{i} \alpha=v_{i}$ and $e_{k} \alpha=v_{k}$ for every $i$ and every $k$. Since $\left\{v_{i}\right\} \dot{\cup}\left\{v_{k}\right\}$ is a basis for $\operatorname{ran} \alpha$, it can be expanded to a basis for $V$, say $\left\{v_{i}\right\} \dot{\cup}\left\{v_{k}\right\} \dot{\cup}\left\{a_{j}\right\} \dot{\cup}\left\{f_{k}\right\}$ (this is possible since $\operatorname{ran} \alpha \cap A=\{0\}$ and $\operatorname{dim} V /(\operatorname{ran} \alpha \oplus A)=|K|)$. Clearly, $\operatorname{dim} V /\left(\operatorname{ran} \alpha^{2} \oplus A\right)=$ $\operatorname{dim}\left\langle v_{k}, f_{k}\right\rangle=2 \xi \neq \xi$, a contradiction. Therefore, $V=\operatorname{ran} \alpha \oplus A$ and $V /(\operatorname{ran} \alpha \oplus A)=$ $\{0\}$. Hence, $V /(\operatorname{ran} \beta \oplus A)=\{0\}$ for every $\beta \in M$, and this implies $V=\operatorname{ran} \beta \oplus A$. Thus, $M \subseteq N(A, \operatorname{dim} A)$ and by the maximality of $M$, we have $M=N(A, \operatorname{dim} A)$, and the result follows.

\section{Acknowledgement}

We thank the referee for several helpful comments on this paper. 


\section{References}

1. A. Cherubini and A. Varisco, Semigroups whose proper subsemigroups are duo, Czechoslovak Math. J., 34(109)(4) (1984), 630-644.

2. A. H. Clifford and G. B. Preston, The Algebraic Theory of Semigroups, Mathematical Surveys, No. 7, Vols. 1 and 2, American Mathematical Society, Providence, RI, 1961 and 1967.

3. J. M. Howie, An introduction to semigroup theory, Academic Press, London, 1976.

4. K. M. Kapp, On bi-ideals and quasi-ideals in semigroups, Publ. Math. Debrecen, 16 (1969), 179-185.

5. Y. Kemprasit, Infinitely many bi-ideals which are not quasi-ideals in some transformation semigroups, Ann. Univ. Sci. Budapest. Eötvös Sect. Math., 44 (2001), 123-128.

6. Y. Kemprasit, Quasi-ideals and bi-ideals in semigroups and rings, pp. 30-46 in Proceedings of the International Conference on Algebra and its Applications (ICAA 2002) (Bangkok), Chulalongkorn Univ., Bangkok, 2002 (available at www.math.sc.chula.ac.th/ICAA2002/main.html).

7. S. Mendes-Gonçalves, Semigroups of injective linear transformations with infinite defect, Comm. Algebra, 34 (1)(2006), 289-302.

8. C. Namnak and Y. Kemprasit, Some semigroups of linear transformations whose sets of bi-ideals and quasi-ideals coincide, pp. 215-224 in Proceedings of the International Conference on Algebra and its Aplications (ICAA2002) (Bangkok), Chulalongkorn Univ., Bangkok, 2002 (available at www.math.sc.chula.ac.th/ICAA2002/main.html).

9. M. A. Reynolds and R. P. Sullivan, Products of idempotent linear transformations, Proc. Royal Soc. Edinburgh, 100A (1985), 123-138.

10. O. Steinfeld, Quasi-ideals in rings and semigroups, Disquisitiones Mathematicae Hungaricae, vol. 10, Akadémiai Kiadó, Budapest, 1978. 